\title{
Delay-Range-Dependent Stability Criteria for Takagi-Sugeno Fuzzy Systems with Fast Time-Varying Delays
}

\author{
Pin-Lin Liu \\ Department of Automation Engineering, Institute of Mechatronoptic System, \\ Chienkuo Technology University, Changhua 500, Taiwan \\ Correspondence should be addressed to Pin-Lin Liu, lpl@ctu.edu.tw
}

Received 23 April 2012; Revised 16 June 2012; Accepted 23 June 2012

Academic Editor: Reinaldo Martinez Palhares

Copyright ( 2012 Pin-Lin Liu. This is an open access article distributed under the Creative Commons Attribution License, which permits unrestricted use, distribution, and reproduction in any medium, provided the original work is properly cited.

\begin{abstract}
The problem of delay-range-dependent stability for T-S fuzzy system with interval time-varying delay is investigated. The constraint on the derivative of the time-varying delay is not required, which allows the time delay to be a fast time-varying function. By developing delay decomposition approach, integral inequalities approach (IIA), and Leibniz-Newton formula, the information of the delayed plant states can be taken into full consideration, and new delay-dependent sufficient stability criteria are obtained in terms of linear matrix inequalities (LMIs) which can be easily solved by various optimization algorithms. Simulation examples show resulting criteria outperform all existing ones in the literature. It is worth pointing out that our criteria are carried out more efficiently for computation and less conservatism of the proposed criteria.
\end{abstract}

\section{Introduction}

It is well known that time delay often appears in the dynamic systems, which is an important source of instability and degradation in the control performance. Fuzzy system in the form of Takagi-Sugeno (T-S) model has been paid considerable attention in the past two decades $[1,2]$. It has been shown that the T-S model method gives an effective way to represent complex nonlinear systems by some simple local linear dynamic systems, and some analysis methods in the linear systems can be effectively extended to the T-S fuzzy systems. However, all the aforementioned criteria aim at time delay free T-S fuzzy systems. In practice, time delay, one of the instability sources in dynamical systems, is a common and complex phenomenon in many industrial and engineering systems such as chemical process, metallurgical processes, biological systems, rolling mill systems, and communication 
networks. As a result, stability analysis for T-S fuzzy systems with time delay is of great significance both in theory and in practice. Some approaches developed for general delay systems have been borrowed to deal with fuzzy systems with time delay. In recent years, the problems of stability and stabilization of the T-S fuzzy systems with time delay have attracted rapidly growing interests [3-21]. Among these references, great efforts have been focused on effective reduction of the conservation of the delayed T-S fuzzy model. Many effective methods, such as new bounding technique for cross-terms [7, 9], augmented Lyapunov functional method [7], and free-weighing matrix method [6, 10,17-20], have been proposed. We can see that the free-weighting matrix approach is used as a main tool to make the criteria less conservative in the literature, and only the lower and upper bounds of delay function $h(t)$ are considered. When stability analysis of delayed systems is concerned, a very effective strategy is to apply the Gu's Lyapunov-Krasovskii functional discretization technique [22]. However, this discretization technique has been developed for linear systems subject to constant time delay. Besides, it is very hard to extend the stability analysis conditions obtained via this technique to control design since several products between decision variables will be generated, leading to nonconvex formulations. Therefore, lessconservative conditions for stability and control of T-S fuzzy systems subject to uncertain time delay are proposed based on a fuzzy weighting-dependent Lyapunov function, the $\mathrm{Gu}$ discretization technique [22], and extra strategies to introduce slack matrix variables by $[13,23]$. However, these results have conservatism to some extent, which exist room for further improvement.

The delay varying in an interval has strong application background, which commonly exists in many practical systems. The investigation for the systems with interval time-varying delay has caused considerable attention, see [13, 18, 24-26] and the references therein. In [15], Lyapunov-Krasovskii function and augmented Lyapunov-Krasovskii function to construct uncorrelated augmented matrix (UAM) and to deal with cross terms in the UAM through improved Jessen's inequality. An improved delay-dependent criterion is derived in [18] by constructing a new Lyapunov functional and using free-weighting matrices. In [24], a weighting delay method is used to deal with the stability of system with time varying delay. In $[25,26]$, by developing a delay decomposition approach, the integral interval $[t-h, t]$ is decomposed into $[t-h, t-\alpha h]$ and $[t-\alpha h, t]$. Since a tuning parameter $\alpha$ is introduced, the information about $x(t-\alpha h)$ can be taken into full consideration; thus, the upper bound of $\int_{t-h}^{t} \dot{x}^{T}(s) R \dot{x}(s) d s$ can be estimated more exactly no matter the delay derivative exists or not. However, it has been realized that too many free variables introduced in the free-weighting matrix method will complicate the system synthesis and consequently lead to a significant computational demand [7]. The problem of improving system performance while reducing the computational demand will be addressed in this paper.

The main contributions of this paper are highlighted as follows. (1) delay-dependent stability criteria are developed, which are an improvement over the latest results available from the open literature $[3,5-7,9,10,12,13,15,17-21,23,27]$; (2) theoretical proof is provided to show that the results in [6] are a special case of the results derived in this paper. The approach developed in this work uses the least number of unknown variables and consequently is the least mathematically complex and most computationally efficient. This implies that some redundant variables in the existing stability criteria can be removed while maintaining the efficiency of the stability conditions. With the present stability conditions, the computational burden is largely reduced; (3) since the delay decomposition approach is introduced in delay interval, it is clear that the stability results are based on the delay decomposition approach. When the positions of delay decomposition are varied, the stability 
results of the proposed criteria are also different. In order to obtain the optimal delay decomposition sequence, we proposed an implementation based on optimization methods.

Motivated by the above discussions, we propose new stability criteria for T-S fuzzy system with interval time-varying delay. By developing a delay decomposition approach, the information of the delayed plant states can be taken into full consideration, and new delay-dependent sufficient stability criteria are obtained in terms of linear matrix inequalities (LMIs) which can be easily solved by various optimization algorithms. Since the delay terms are concerned more exactly, less conservative results are presented. Moreover, the restriction on the change rate of time-varying delays is relaxed in the proposed criteria. The proposed stability conditions are much less conservative and are more general than some existing results. Numerical examples are given to illustrate the effectiveness of our theoretical results.

\section{Stability Analysis}

Consider a T-S fuzzy system with a time-varying delay, which is represented by a T-S fuzzy model, composed of a set of fuzzy implications, and each implication is expressed by a linear system model. The $i$ th rule of this T-S fuzzy model is of the following form:

Plant rule $i$ : if $z_{1}(t)$ is $M_{i 1}$, and $\ldots$, and $z_{p}(t)$ is $M_{i p}$ then

$$
\begin{gathered}
\dot{x}(t)=A_{i} x(t)+A_{\mathrm{di}} x(t-h(t)), \\
x(t)=\phi(t), \quad t \in[-h, 0], \quad i=1,2, \ldots, r,
\end{gathered}
$$

where $z_{1}(t), z_{2}(t), \ldots, z_{p}(t)$ are the premise variables; $M_{i j}, i=1,2, \ldots, r, j=1,2, \ldots, p$ are the fuzzy sets; $x(t) \in R^{n}$ is the state; $\phi(t)$ is a vector-valued initial condition; $A_{i}$ and $A_{\mathrm{di}}$ are constant real matrices with appropriate dimensions; the scalars ris the number of if-then rules; time delay, $h(t)$, is a time-varying delay. We will consider the following two cases for the timevarying delay.

Case 1. $h(t)$ is a differentiable function satisfying

$$
0 \leq h_{1} \leq h(t) \leq h_{2}, \quad|\dot{h}(t)| \leq h_{d}, \quad \forall t \geq 0 .
$$

Case 2. $h(t)$ is a differentiable function satisfying

$$
0 \leq h_{1} \leq h(t) \leq h_{2}
$$

where $h_{1}$ and $h_{2}$ are the lower and upper delay bounds, respectively; $h_{1}, h_{2}$, and $h_{d}$ are constants. Here $h_{1}$ the lower bound of delay may not be equal to 0 , and when $h_{d}=0$ we have $h_{1}=h_{2}$. Both Cases 1 and 2 have considered the upper and nonzero lower delay bounds of the interval time-varying delay. Case 1 is a special case of Case 2. If the time-varying delay is differentiable and $h_{d}<1$, one can obtain a less conservative result using Case 1 than that using Case 2. 
By fuzzy blending, the overall fuzzy model is inferred as follows:

$$
\begin{aligned}
\dot{x}(t) & =\frac{\sum_{i=1}^{r} w_{i}(z(t))\left[A_{i} x(t)+A_{\mathrm{di}} x(t-h(t))\right]}{\sum_{i=1}^{r} w_{i}(z(t))} \\
& =\sum_{i=1}^{r} \theta_{i}(z(t))\left[A_{i} x(t)+A_{\mathrm{di}} x(t-h(t))\right] \\
& =A x(t)+A_{d} x(t-h(t)) \\
& x(t)=\sum_{i=1}^{r} \theta_{i}(z(t)) \phi_{i}(t), \quad t \in[-h, 0],
\end{aligned}
$$

where $z=\left[z_{1}, z_{2}, \ldots, z_{p}\right] ; w_{i}: R^{p} \rightarrow[0,1], i=1,2, \ldots, r$, is the membership function of the system with respect to the plant rule $i ; \theta_{i}(z(t))=w_{i}(z(t)) / \sum_{i=1}^{r} w_{i}(z(t)) ; A=$ $\sum_{i=1}^{r} \theta_{i}(z(t)) A_{i}, A_{d}=\sum_{i=1}^{r} \theta_{i}(z(t)) A_{\mathrm{di}}$, and It is assumed that $w_{i}(z(t)) \geq 0, i=$ $1,2, \ldots, r, \sum_{i=1}^{r} w_{i}(z(t)) \geq 0$ for all $t$, so we have $\theta_{i}(z(t)) \geq 0, \sum_{i=1}^{r} \theta_{i}(z(t))=1$.

In the following, we will develop some practically computable stability criteria for the system described (2.1a). The following lemmas are useful in deriving the criteria. First, we introduce the following technical Lemma 2.1 of integral inequality approach (IIA).

Lemma 2.1 (see [11]). For any positive semidefinite matrices

$$
X=\left[\begin{array}{lll}
X_{11} & X_{12} & X_{13} \\
X_{12}^{T} & X_{22} & X_{23} \\
X_{13}^{T} & X_{23}^{T} & X_{33}
\end{array}\right] \geq 0
$$

Then, one obtains

$$
-\int_{t-h}^{t} \dot{x}^{T}(s) X_{33} \dot{x}(s) d s \leq \int_{t-h}^{t}\left[\begin{array}{lll}
x^{T}(t) & x^{T}(t-h) & \dot{x}^{T}(s)
\end{array}\right]\left[\begin{array}{ccc}
X_{11} & X_{12} & X_{13} \\
X_{12}^{T} & X_{22} & X_{23} \\
X_{13}^{T} & X_{23}^{T} & 0
\end{array}\right]\left[\begin{array}{c}
x(t) \\
x(t-h) \\
\dot{x}(s)
\end{array}\right] d s .
$$

Lemma 2.2 (see [28]). The following matrix inequality

$$
\left[\begin{array}{cc}
Q(x) & S(x) \\
S^{T}(x) & R(x)
\end{array}\right]<0
$$

where $Q(x)=Q^{T}(x), R(x)=R^{T}(x)$ and $S(x)$ depend on affine on $x$ is equivalent to

$$
\begin{gathered}
R(x)<0, \\
Q(x)<0, \\
Q(x)-S(x) R^{-1}(x) S^{T}(x)<0 .
\end{gathered}
$$


In this paper, a new Lyapunov functional is constructed, which contains the information of the lower bound of delay $h_{1}$ and upper bound $h_{2}$.The following Theorem 2.3 presents a delay-range-dependent result in terms of LMIs and expresses the relationships between the terms of the Leibniz-Newton formula.

Theorem 2.3. Under Case 1, for given scalars $h_{1}, h_{2}, h_{d}$, and $\alpha(0<\alpha<1)$, System (2.4) subject to (2.2) is asymptotically stable if there exist symmetry positive-definite matrices $P=P^{T}>0, Q_{1}=$ $Q_{1}^{T}>0, Q_{2}=Q_{2}^{T}>0, Q_{3}=Q_{3}^{T}>0, R_{1}=R_{1}^{T}>0, R_{2}=R_{2}^{T}>0$, and positive semidefinite matrices

$$
X=\left[\begin{array}{lll}
X_{11} & X_{12} & X_{13} \\
X_{12}^{T} & X_{22} & X_{23} \\
X_{13}^{T} & X_{23}^{T} & X_{33}
\end{array}\right] \geq 0, \quad Y=\left[\begin{array}{lll}
Y_{11} & Y_{12} & Y_{13} \\
Y_{12}^{T} & Y_{22} & Y_{23} \\
Y_{13}^{T} & Y_{23}^{T} & Y_{33}
\end{array}\right] \geq 0, \quad Z=\left[\begin{array}{lll}
Z_{11} & Z_{12} & Z_{13} \\
Z_{12}^{T} & Z_{22} & Z_{23} \\
Z_{13}^{T} & Z_{23}^{T} & Z_{33}
\end{array}\right] \geq 0
$$

such that the following LMIs hold:

$$
\begin{gathered}
\Xi_{i}=\left[\begin{array}{cccccc}
\Xi_{11} & \Xi_{12} & 0 & \Xi_{14} & \Xi_{15} & \Xi_{16} \\
\Xi_{12}^{T} & \Xi_{22} & \Xi_{23} & \Xi_{24} & \Xi_{25} & \Xi_{26} \\
0 & \Xi_{23}^{T} & \Xi_{33} & 0 & 0 & 0 \\
\Xi_{14}^{T} & \Xi_{24}^{T} & 0 & \Xi_{44} & 0 & 0 \\
\Xi_{15}^{T} & \Xi_{25}^{T} & 0 & 0 & \Xi_{55} & 0 \\
\Xi_{16}^{T} & \Xi_{26}^{T} & 0 & 0 & 0 & \Xi_{66}
\end{array}\right]<0 \\
R_{1}-X_{33} \geq 0 \\
R_{2}-Y_{33} \geq 0 \\
R_{2}-Z_{33} \geq 0
\end{gathered}
$$

where

$$
\begin{aligned}
& \Xi_{11}=A_{i}^{T} P+P A_{i}+Q_{1}+Q_{2}+Q_{3}+\alpha \delta X_{11}+X_{13}+X_{13}^{T}, \\
& \Xi_{12}=P A_{d i}, \quad \Xi_{14}=\alpha \delta X_{12}-X_{13}+X_{23}^{T}, \\
& \Xi_{15}=\alpha \delta A_{i}^{T} R_{1}, \quad \Xi_{16}=\left(h_{2}-\alpha \delta\right) A_{i}^{T} R_{2}, \\
& \Xi_{22}=-\left(1-h_{d}\right) Q_{2}+\left(h_{2}-\alpha \delta\right) Y_{22}-Y_{23}-Y_{23}^{T}+\left(h_{2}-\alpha \delta\right) Z_{11}+Z_{13}+Z_{13}^{T}, \\
& \Xi_{23}=\left(h_{2}-\alpha \delta\right) Z_{12}-Z_{13}+Z_{23}^{T}, \quad \Xi_{24}=\left(h_{2}-\alpha \delta\right) Y_{12}^{T}-Y_{13}^{T}+Y_{23}, \quad \Xi_{25}=\alpha \delta A_{d i}^{T} R_{1}, \\
& \Xi_{26}=\left(h_{2}-\alpha \delta\right) A_{d i}^{T} R_{2}, \quad \Xi_{33}=-Q_{3}+\left(h_{2}-\alpha \delta\right) Z_{22}-Z_{23}-Z_{23}^{T}, \\
& \Xi_{44}=-Q_{1}+\left(h_{2}-\alpha \delta\right) Y_{11}+Y_{13}+Y_{13}^{T}+\alpha \delta X_{22}-X_{23}-X_{23}^{T}, \\
& \Xi_{55}=-\delta R_{1}, \quad \Xi_{66}=-\left(h_{2}-\alpha \delta\right) R_{2} .
\end{aligned}
$$

Proof. If we can proof that Theorem 2.3 holds for two cases, that is, $\alpha \delta \leq h(t) \leq h_{2}$ and $h_{1} \leq h(t) \leq \alpha \delta$, where $\alpha \delta=\left(h_{2}+h_{1}\right) / 2$, then Theorem 2.3 is true. 
Case 1. When $\alpha \delta \leq h(t) \leq h_{2}$.

Construct a Lyapunov-Krasovskii functional candidate as

$$
\begin{aligned}
V\left(x_{t}\right)= & x^{T}(t) P x(t)+\int_{t-\alpha \delta}^{t} x^{T}(s) Q_{1} x(s) d s+\int_{t-h(t)}^{t} x^{T}(s) Q_{2} x(s) d s+\int_{t-h_{2}}^{t} x^{T}(s) Q_{3} x(s) d s \\
& +\int_{-\alpha \delta}^{0} \int_{t+\theta}^{t} \dot{x}^{T}(s) R_{1} \dot{x}(s) d s d \theta+\int_{-h_{2}}^{-\alpha \delta} \int_{t+\theta}^{t} \dot{x}^{T}(s) R_{2} \dot{x}(s) d s d \theta .
\end{aligned}
$$

Calculating the derivative of (2.12) with respect to $t>0$ along the trajectories of (2.1a) and $(2.1 \mathrm{~b})$ leads to

$$
\begin{aligned}
\dot{V}\left(x_{t}\right)= & x^{T}(t)\left(P A+A^{T} P\right) x(t)+x^{T}(t) P A_{d} x(t-h(t))+x^{T}(t-h(t)) A_{d}^{T} P x(t) \\
& +x^{T}(t)\left(Q_{1}+Q_{2}+Q_{3}\right) x(t)-x^{T}(t-\alpha \delta) Q_{1} x(t-\alpha \delta) \\
& -x^{T}(t-h(t))(1-\dot{h}(t)) Q_{2} x(t-h(t))-x^{T}\left(t-h_{2}\right) Q_{3} x\left(t-h_{2}\right)+\dot{x}^{T}(t) \alpha \delta R_{1} \dot{x}(t) \\
& +\dot{x}^{T}(t)\left(h_{2}-\alpha \delta\right) R_{2} \dot{x}(t)-\int_{t-\delta}^{t} \dot{x}^{T}(s) R_{1} \dot{x}(s) d s-\int_{t-h_{2}}^{t-\delta} \dot{x}^{T}(s) R_{2} \dot{x}(s) d s \\
\leq & x^{T}(t)\left(P A+A^{T} P\right) x(t)+x^{T}(t) P A_{d} x(t-h(t))+x^{T}(t-h(t)) A_{d}^{T} P x(t) \\
& +x^{T}(t)\left(Q_{1}+Q_{2}+Q_{3}\right) x(t)-x^{T}(t-\alpha \delta) Q_{1} x(t-\alpha \delta) \\
& -x^{T}(t-h(t))\left(1-h_{d}\right) Q_{2} x(t-h(t))-x^{T}\left(t-h_{2}\right) Q_{3} x\left(t-h_{2}\right) \\
& +\dot{x}^{T}(t)\left[\alpha \delta R_{1}+\left(h_{2}-\alpha \delta\right) R_{2}\right] \dot{x}(t)-\int_{t-\alpha \delta}^{t} \dot{x}^{T}(s) R_{1} \dot{x}(s) d s-\int_{t-h_{2}}^{t-\alpha \delta} \dot{x}^{T}(s) R_{2} \dot{x}(s) d s,
\end{aligned}
$$

with the operator for the term $\dot{x}^{T}(t)\left[\alpha \delta R_{1}+\left(h_{2}-\alpha \delta\right) R_{2}\right] \dot{x}(t)$ as follows:

$$
\begin{aligned}
\dot{x}^{T}(t) & {\left[\alpha \delta R_{1}+\left(h_{2}-\alpha \delta\right) R_{2}\right] \dot{x}(t) } \\
= & {\left[A x(t)+A_{d} x(t-h(t))\right]^{T}\left[\alpha \delta R_{1}+\left(h_{2}-\alpha \delta\right) R_{2}\right]\left[A x(t)+A_{d} x(t-h(t))\right] } \\
= & x^{T}(t) A^{T}\left[\alpha \delta R_{1}+\left(h_{2}-\alpha \delta\right) R_{2}\right] A x(t)+x^{T}(t) A^{T}\left[\alpha \delta R_{1}+\left(h_{2}-\alpha \delta\right) R_{2}\right] A_{d} x(t-h(t)) \\
& +x^{T}(t-h(t)) A_{d}^{T}\left[\alpha \delta R_{1}+\left(h_{2}-\alpha \delta\right) R_{2}\right] A x(t) \\
& +x^{T}(t-h(t)) A_{d}^{T}\left[\alpha \delta R_{1}+\left(h_{2}-\alpha \delta\right) R_{2}\right] A_{d} x(t-h(t)) .
\end{aligned}
$$


Alternatively, the following equations are true:

$$
\begin{aligned}
-\int_{t-\alpha \delta}^{t} & \dot{x}^{T}(s) R_{1} \dot{x}(s) d s-\int_{t-h_{2}}^{t-\alpha \delta} \dot{x}^{T}(s) R_{2} \dot{x}(s) d s \\
= & -\int_{t-\alpha \delta}^{t} \dot{x}^{T}(s) R_{1} \dot{x}(s) d s-\int_{t-h(t)}^{t-\alpha \delta} \dot{x}^{T}(s) R_{2} \dot{x}(s) d s-\int_{t-h_{2}}^{t-h(t)} \dot{x}^{T}(s) R_{2} \dot{x}(s) d s \\
= & -\int_{t-\alpha \delta}^{t} \dot{x}^{T}(s)\left(R_{1}-X_{33}\right) \dot{x}(s) d s-\int_{t-h(t)}^{t-\alpha \delta} \dot{x}^{T}(s)\left(R_{2}-Y_{33}\right) \dot{x}(s) d s \\
& -\int_{t-h_{2}}^{t-h(t)} \dot{x}^{T}(s)\left(R_{2}-Z_{33}\right) \dot{x}(s) d s-\int_{t-\alpha \delta}^{t} \dot{x}^{T}(s) X_{33} \dot{x}(s) d s \\
& -\int_{t-h(t)}^{t-\alpha \delta} \dot{x}^{T}(s) Y_{33} \dot{x}(s) d s-\int_{t-h_{2}}^{t-h(t)} \dot{x}^{T}(s) Z_{33} \dot{x}(s) d s .
\end{aligned}
$$

By utilizing Lemma 2.1 and the Leibniz-Newton formula, we have

$$
\begin{aligned}
& -\int_{t-\alpha \delta}^{t} \dot{x}^{T}(s) X_{33} \dot{x}(s) d s \\
& \leq \int_{t-\alpha \delta}^{t}\left[\begin{array}{lll}
x^{T}(t) & x^{T}(t-\alpha \delta) & \dot{x}^{T}(s)
\end{array}\left[\begin{array}{lll}
X_{11} & X_{12} & X_{13} \\
X_{12}^{T} & X_{22} & X_{23} \\
X_{13}^{T} & X_{23}^{T} & 0
\end{array}\right]\left[\begin{array}{c}
x(t) \\
x(t-\alpha \delta) \\
\dot{x}(s)
\end{array}\right] d s\right. \\
& \leq x^{T}(t) \alpha \delta X_{11} x(t)+x^{T}(t) \alpha \delta X_{12} x(t-\alpha \delta)+x^{T}(t-\alpha \delta) \alpha \delta X_{12}^{T} x(t) \\
& +x^{T}(t-\alpha \delta) \alpha \delta X_{22} x(t-\alpha \delta)+x^{T}(t) X_{13}^{T} \int_{t-\alpha \delta}^{t} \dot{x}(s) d s+x^{T}(t-\alpha \delta) X_{23}^{T} \int_{t-\alpha \delta}^{t} \dot{x}(s) d s \\
& +\int_{t-\alpha \delta}^{t} \dot{x}^{T}(s) d s X_{13} x(t)+\int_{t-\alpha \delta}^{t} \dot{x}^{T}(s) d s X_{23} x(t-\alpha \delta) \\
& =x^{T}(t)\left[\alpha \delta X_{11}+X_{13}^{T}+X_{13}\right] x(t)+x^{T}(t)\left[\alpha \delta X_{12}-X_{13}+X_{23}^{T}\right] x(t-\alpha \delta) \\
& +x^{T}(t-\alpha \delta)\left[\delta X_{12}^{T}+X_{23}-X_{13}^{T}\right] x(t)+x^{T}(t-\alpha \delta)\left[\alpha \delta X_{22}-X_{23}-X_{23}^{T}\right] x(t-\alpha \delta) .
\end{aligned}
$$


Similarly, we obtain

$$
\begin{aligned}
& -\int_{t-h(t)}^{t-\alpha \delta} \dot{x}^{T}(s) Y_{33} \dot{x}(s) d s \\
& \leq x^{T}(t-\alpha \delta)\left[\left(h_{2}-\alpha \delta\right) Y_{11}+Y_{13}^{T}+Y_{13}\right] x(t-\alpha \delta) \\
& \quad+x^{T}(t-\alpha \delta)\left[\left(h_{2}-\alpha \delta\right) Y_{12}-Y_{13}+Y_{23}^{T}\right] x(t-h(t)) \\
& \quad+x^{T}(t-h(t))\left[\left(h_{2}-\alpha \delta\right) Y_{12}^{T}-Y_{13}^{T}+Y_{23}\right] x(t-\alpha \delta) \\
& \quad+x^{T}(t-h(t))\left[\left(h_{2}-\alpha \delta\right) Y_{22}-Y_{23}-Y_{23}^{T}\right] x(t-h(t)) \\
& -\int_{t-}^{t-} h_{2}(t) \dot{x}^{T}(s) Z_{33} \dot{x}(s) d s \\
& \leq x^{T}(t-h(t))\left[\left(h_{2}-\alpha \delta\right) Z_{11}+Z_{13}+Z_{13}^{T}\right] x(t-h(t)) \\
& \quad+x^{T}(t-h(t))\left[\left(h_{2}-\alpha \delta\right) Z_{12}-Z_{13}+Z_{23}^{T}\right] x\left(t-h_{2}\right) \\
& \quad+x^{T}\left(t-h_{2}\right)\left[\left(h_{2}-\alpha \delta\right) Z_{12}^{T}-Z_{13}^{T}+Z_{23}\right] x(t-h(t)) \\
& \quad+x^{T}\left(t-h_{2}\right)\left[\left(h_{2}-\alpha \delta\right) Z_{22}-Z_{23}-Z_{23}^{T}\right] x\left(t-h_{2}\right) .
\end{aligned}
$$

Substituting the above equations (2.14)-(2.18) into (2.13), we obtain

$$
\begin{aligned}
\dot{V}\left(x_{t}\right) \leq & \xi^{T}(t) \Omega \xi(t)-\int_{t-\alpha \delta}^{t} \dot{x}^{T}(s)\left(R_{1}-X_{33}\right) \dot{x}(s) d s-\int_{t-h(t)}^{t-\alpha \delta} \dot{x}^{T}(s)\left(R_{2}-Y_{33}\right) \dot{x}(s) d s \\
& -\int_{t-h_{2}}^{t-h(t)} \dot{x}^{T}(s)\left(R_{2}-Z_{33}\right) \dot{x}(s) d s
\end{aligned}
$$

where $\xi^{T}(t)=\left[\begin{array}{llll}x^{T}(t) & x^{T}(t-h(t)) & x^{T}\left(t-h_{2}\right) & x^{T}(t-\alpha \delta)\end{array}\right]$ and

$$
\Omega=\left[\begin{array}{cccc}
\Omega_{11} & \Omega_{12} & 0 & \Omega_{14} \\
\Omega_{12}^{T} & \Omega_{22} & \Omega_{23} & \Omega_{24} \\
0 & \Omega_{23}^{T} & \Omega_{33} & 0 \\
\Omega_{14}^{T} & \Omega_{24}^{T} & 0 & \Omega_{44}
\end{array}\right],
$$


where

$$
\begin{aligned}
\Omega_{11}= & A^{T} P+P A+Q_{1}+Q_{2}+Q_{3}+\alpha \delta X_{11}+X_{13}+X_{13}^{T}+A^{T}\left[\alpha \delta R_{1}+\left(h_{2}-\alpha \delta\right) R_{2}\right] A, \\
\Omega_{12}= & P A_{d}+A^{T}\left[\alpha \delta R_{1}+\left(h_{2}-\alpha \delta\right) R_{2}\right] A_{d}, \\
\Omega_{14}= & \alpha \delta X_{12}-X_{13}+X_{23}^{T}, \\
\Omega_{22}= & -\left(1-h_{d}\right) Q_{2}+\left(h_{2}-\alpha \delta\right) Z_{11}+Z_{13}+Z_{13}^{T}+\left(h_{2}-\alpha \delta\right) Y_{22}-Y_{23}-Y_{23}^{T} \\
& +A_{d}^{T}\left[\alpha \delta R_{1}+\left(h_{2}-\alpha \delta\right) R_{2}\right] A_{d}, \\
\Omega_{23}= & \left(h_{2}-\alpha \delta\right) Z_{12}-Z_{13}+Z_{23}^{T}, \\
\Omega_{24}= & \left(h_{2}-\alpha \delta\right) Y_{12}^{T}-Y_{13}^{T}+Y_{23}, \\
\Omega_{33}= & -Q_{3}+\left(h_{2}-\alpha \delta\right) Z_{22}-Z_{23}-Z_{23}^{T}, \\
\Omega_{44}= & -Q_{1}+\left(h_{2}-\alpha \delta\right) Y_{11}+Y_{13}+Y_{13}^{T}+\alpha \delta X_{22}-X_{23}-X_{23}^{T} .
\end{aligned}
$$

If $\Omega<0, R_{1}-X_{33} \geq 0, R_{2}-Y_{33} \geq 0$, and $R_{2}-Z_{33} \geq 0$, then $\dot{V}\left(x_{t}\right)<-\varepsilon\|x(t)\|^{2}$ for a sufficiently small $\varepsilon>0$. By the Schur complement of Lemma 2.2, $\Omega<0$ is equivalent to the following inequality and is true:

$$
\Psi=\left[\begin{array}{cccccc}
\Psi_{11} & \Psi_{12} & 0 & \Psi_{14} & \Psi_{15} & \Psi_{16} \\
\Psi_{12}^{T} & \Psi_{22} & \Psi_{23} & \Psi_{24} & \Psi_{25} & \Psi_{26} \\
0 & \Psi_{23}^{T} & \Psi_{33} & 0 & 0 & 0 \\
\Psi_{14}^{T} & \Psi_{24}^{T} & 0 & \Psi_{44} & 0 & 0 \\
\Psi_{15}^{T} & \Psi_{25}^{T} & 0 & 0 & \Psi_{55} & 0 \\
\Psi_{16}^{T} & \Psi_{26}^{T} & 0 & 0 & 0 & \Psi_{66}
\end{array}\right]<0
$$

where

$$
\begin{aligned}
& \Psi_{11}=A^{T} P+P A+Q_{1}+Q_{2}+Q_{3}+\alpha \delta X_{11}+X_{13}+X_{13}^{T}, \quad \Psi_{12}=P A_{d}, \\
& \Psi_{14}=\alpha \delta X_{12}-X_{13}+X_{23}^{T}, \quad \Psi_{15}=\alpha \delta A^{T} R_{1}, \quad \Psi_{16}=\left(h_{2}-\alpha \delta\right) A^{T} R_{2}, \\
& \Psi_{22}=-\left(1-h_{d}\right) Q_{2}+\left(h_{2}-\alpha \delta\right) Y_{22}-Y_{23}-Y_{23}^{T}+\left(h_{2}-\alpha \delta\right) Z_{11}+Z_{13}+Z_{13}^{T}, \\
& \Psi_{23}=\left(h_{2}-\alpha \delta\right) Z_{12}-Z_{13}+Z_{23}^{T}, \quad \Psi_{24}=\left(h_{2}-\alpha \delta\right) Y_{12}^{T}-Y_{13}^{T}+Y_{23}, \quad \Psi_{25}=\alpha \delta A_{d}^{T} R_{1}, \\
& \Psi_{26}=\left(h_{2}-\alpha \delta\right) A_{d}^{T} R_{2}, \quad \Psi_{33}=-Q_{3}+\left(h_{2}-\alpha \delta\right) Z_{22}-Z_{23}-Z_{23}^{T}, \\
& \Psi_{44}=-Q_{1}+\left(h_{2}-\alpha \delta\right) Y_{11}+Y_{13}+Y_{13}^{T}+\alpha \delta X_{22}-X_{23}-X_{23}^{T}, \\
& \Psi_{55}=-\alpha \delta R_{1}, \quad \Psi_{66}=-\left(h_{2}-\alpha \delta\right) R_{2} .
\end{aligned}
$$

That is to say, if $\Psi<0, R_{1}-X_{33} \geq 0, R_{2}-Y_{33} \geq 0$, and $R_{2}-Z_{33} \geq 0$, then $\dot{V}(t)<-\varepsilon\|x(t)\|^{2}$ for a sufficiently small $\varepsilon>0$. Furthermore, (2.10a) implies $\sum_{i=1}^{r} \theta_{i}(z(t)) \Omega_{i}<0$, which is 
equivalent to (2.22). Therefore, if LMIs (2.10a) are feasible, the system (2.4) is asymptotically stable.

Case 2. When $h_{1} \leq h(t) \leq \alpha \delta$.

For this case, the Lyapunov-Krasovskii functional candidate is chosen

$$
\begin{aligned}
V\left(x_{t}\right)= & x^{T}(t) P x(t)+\int_{t-\alpha \delta}^{t} x^{T}(s) Q_{1} x(s) d s+\int_{t-h(t)}^{t} x^{T}(s) Q_{2} x(s) d s+\int_{t-h_{1}}^{t} x^{T}(s) Q_{3} x(s) d s \\
& +\int_{-\alpha \delta}^{0} \int_{t+\theta}^{t} \dot{x}^{T}(s) R_{1} \dot{x}(s) d s d \theta+\int_{-\alpha \delta}^{-h_{1}} \int_{t+\theta}^{t} \dot{x}^{T}(s) R_{2} \dot{x}(s) d s d \theta
\end{aligned}
$$

where $P>0, R_{1}>0, R_{2}>0, Q_{i}>0(i=1,2,3)$.

Choosing $\xi^{T}(t)=\left[\begin{array}{llll}x^{T}(t) & x^{T}(t-h(t)) & x^{T}\left(t-h_{1}\right) & x^{T}(t-\alpha \delta)\end{array}\right]$ and then using a proof process similar to that for Case 1 , we derive the same condition (2.10a), (2.10b), (2.10c), and (2.10d) as that for Case 1 . This completes the proof.

When the information of the time derivative of delay is unknown, by eliminating $Q_{2}$ we have the following result from Theorem 2.3.

Corollary 2.4. For given scalars $h_{1}, h_{2}$, and $\alpha(0<\alpha<1)$, the system (2.4) is asymptotically stable if there exist positive-definite matrices $P=P^{T}>0, Q_{1}=Q_{1}^{T}>0, Q_{3}=Q_{3}^{T}>0, R_{1}=R_{1}^{T}>0$, $R_{2}=R_{2}^{T}>0$ and positive semidefinite matrices

$$
X=\left[\begin{array}{lll}
X_{11} & X_{12} & X_{13} \\
X_{12}^{T} & X_{22} & X_{23} \\
X_{13}^{T} & X_{23}^{T} & X_{33}
\end{array}\right] \geq 0, \quad Y=\left[\begin{array}{lll}
Y_{11} & Y_{12} & Y_{13} \\
Y_{12}^{T} & Y_{22} & Y_{23} \\
Y_{13}^{T} & Y_{23}^{T} & Y_{33}
\end{array}\right] \geq 0, \quad Z=\left[\begin{array}{lll}
Z_{11} & Z_{12} & Z_{13} \\
Z_{12}^{T} & Z_{22} & Z_{23} \\
Z_{13}^{T} & Z_{23}^{T} & Z_{33}
\end{array}\right] \geq 0
$$

such that the following LMIs hold:

$$
\bar{\Xi}_{i}=\left[\begin{array}{cccccc}
\Xi_{11} & \Xi_{12} & 0 & \Xi_{14} & \Xi_{15} & \Xi_{16} \\
\Xi_{12}^{T} & \bar{\Xi}_{22} & \Xi_{23} & \Xi_{24} & \Xi_{25} & \Xi_{26} \\
0 & \Xi_{23}^{T} & \Xi_{33} & 0 & 0 & 0 \\
\Xi_{14}^{T} & \Xi_{24}^{T} & 0 & \Xi_{44} & 0 & 0 \\
\Xi_{15}^{T} & \Xi_{25}^{T} & 0 & 0 & \Xi_{55} & 0 \\
\Xi_{16}^{T} & \Xi_{26}^{T} & 0 & 0 & 0 & \Xi_{66}
\end{array}\right]<0
$$


Journal of Applied Mathematics

$$
\begin{aligned}
& R_{2}-Y_{33} \geq 0, \\
& R_{2}-Z_{33} \geq 0,
\end{aligned}
$$

where $\Xi_{i j}(i, j=1,2, \ldots, 6)$ are defined in (8) and

$$
\begin{aligned}
& \bar{\Xi}_{11}=A^{T} P+P A+Q_{1}+Q_{3}+\alpha \delta X_{11}+X_{13}+X_{13}^{T} \\
& \bar{\Xi}_{22}=\left(h_{2}-\alpha \delta\right) Y_{22}-Y_{23}-Y_{23}^{T}+\left(h_{2}-\alpha \delta\right) Z_{11}+Z_{13}+Z_{13}^{T} .
\end{aligned}
$$

Proof. If the matrix $Q_{2}=0$ is selected in (2.10a), (2.10b), (2.10c), and (2.10d), this proof can be completed in a similar formulation to Theorem 2.3.

When $h_{1}=0$, Theorem 2.3 reduces to the following Corollary 2.5 .

Corollary 2.5. For given scalars $h, h_{d}$, and $\alpha(0<\alpha<1)$, the system (2.4) is asymptotically stable if there exist symmetry positive-definite matrices $P=P^{T}>0, Q_{1}=Q_{1}^{T}>0, Q_{2}=Q_{2}^{T}>0, R=R^{T}>0$, and positive semidefinite matrices

$$
X=\left[\begin{array}{lll}
X_{11} & X_{12} & X_{13} \\
X_{12}^{T} & X_{22} & X_{23} \\
X_{13}^{T} & X_{23}^{T} & X_{33}
\end{array}\right] \geq 0, \quad Y=\left[\begin{array}{lll}
Y_{11} & Y_{12} & Y_{13} \\
Y_{12}^{T} & Y_{22} & Y_{23} \\
Y_{13}^{T} & Y_{23}^{T} & Y_{33}
\end{array}\right] \geq 0
$$

such that the following LMIs hold for $i=1,2, \ldots, r$,

$$
\begin{aligned}
& \Sigma_{i}=\left[\begin{array}{cccc}
\Sigma_{11} & \Sigma_{12} & 0 & \Sigma_{14} \\
\Sigma_{12}^{T} & \Sigma_{22} & \Sigma_{23} & \Sigma_{24} \\
0 & \Sigma_{23}^{T} & \Sigma_{33} & 0 \\
\Sigma_{14}^{T} & \Sigma_{24}^{T} & 0 & \Sigma_{44}
\end{array}\right]<0, \\
& R-X_{33} \geq 0, \\
& R-Y_{33} \geq 0 \text {, }
\end{aligned}
$$

where

$$
\begin{aligned}
& \Sigma_{11}=A_{i}^{T} P+P A_{i}+Q_{1}+Q_{2}+\alpha h X_{11}+X_{13}+X_{13}^{T}, \quad \Sigma_{12}=P A_{d i}+\alpha h X_{12}-X_{13}+X_{23}^{T}, \\
& \Sigma_{14}=\alpha h A_{i}^{T} R, \quad \Sigma_{22}=-\left(1-h_{d}\right) Q_{2}+\alpha h X_{22}-X_{23}-X_{23}^{T}+h Y_{11}+Y_{13}+Y_{13}^{T}, \\
& \Sigma_{23}=\alpha h Y_{12}-Y_{13}+Y_{23}^{T}, \quad \Sigma_{24}=\alpha h A_{d i}^{T} R, \\
& \Sigma_{33}=-Q_{1}+\alpha h Y_{22}-Y_{23}-Y_{23}^{T}, \quad \Omega_{44}=-\alpha h R .
\end{aligned}
$$

Proof. Choose the following fuzzy Lyapunov-Krasovskii functional candidate to be

$$
V(t)=V_{1}(t)+V_{2}(t)+V_{3}(t)+V_{4}(t),
$$


where

$$
\begin{aligned}
& V_{1}(t)=x^{T}(t) \operatorname{Px}(t) \\
& V_{2}(t)=\int_{t-\alpha h}^{t} x^{T}(s) Q_{1} x(s) d s \\
& V_{3}(t)=\int_{t-h(t)}^{t} x^{T}(s) Q_{2} x(s) d s \\
& V_{4}(t)=\int_{-\alpha h}^{0} \int_{t+\theta}^{t} \dot{x}^{T}(s) R \dot{x}(s) d s d \theta .
\end{aligned}
$$

Then, taking the time derivative of $V(t)$ with respect to $t$ along the system (2.4) yield

$$
\dot{V}(t)=\dot{V}_{1}(t)+\dot{V}_{2}(t)+\dot{V}_{3}(t)+\dot{V}_{4}(t) .
$$

Then the proof follows a linear similar to the proof of Theorem 2.3 and thus is omitted here.

Remark 2.6. In our Theorems $2.3, h_{d}$ can be any value or unknown due to $\Xi_{22}=-\left(1-h_{d}\right) Q_{2}+$ $\left(h_{2}-\alpha \delta\right) Y_{22}-Y_{23}-Y_{23}^{T}+\left(h_{2}-\alpha \delta\right) Z_{11}+Z_{13}+Z_{13}^{T}$. Therefore, Theorem 2.3 is applicable to both cases of fast and slow time varying delay. We will show the other characteristic of Theorem 2.3. When the distance between $h_{1}$ and $h_{2}$ is sufficiently small, the upper bound $h_{2}$ of delay for unknown $h_{d}$ will be very close to the upper bound for $h_{d}=0$. This characteristic is not included in previous Lyapunov functional based work where the upper bound of delay for $h_{d} \neq 0$ is always less than that for $h_{d}=0$.

Remark 2.7. It is seen from the proof of Theorem 2.3 and Corollary 2.4 that the main characteristics of the method developed in this paper can be generalized as the following two steps. (i) Construct a Lyapunov function to integrated both lower and upper delay bounds, for example, $\alpha \delta=\left(h_{2}+h_{1}\right) / 2$ in (2.12) and (2.24). (ii) Employ Lemma 2.1 to deal with cross-product terms, for example, those in (2.15)-(2.18). It is also seen from the proof that neither model transformation nor free-weighting matrices have been employed to deal with the cross-product terms. Therefore, the stability criteria derived in this paper are expected to be less conservative. This will be demonstrated later through numerical examples. It is noted that although it has been observed that using $\alpha \delta=\left(h_{2}+h_{1}\right) / 2$ in the constructed Lyapunov function can improve stability performance for many examples, theoretical evidence has not been found so far to explain the observations.

Based on that, a convex optimization problem is formulated to find the bound on the allowable delay time $0 \leq h_{1} \leq h(t) \leq h_{2}$ which maintains the delay-dependent stability of the time delay system (2.4).

Remark 2.8. It is interesting to note that $h_{1}, h_{2}$ appears linearly in (2.10a) and (2.26a). Thus, a generalized eigenvalue problem (GEVP) as defined in Boyd et al. [28] can be formulated to solve the minimum acceptable $1 / h_{1}$ (or $1 / h_{2}$ ) and, therefore, the maximum $h_{1}$ (or $h_{2}$ ) to maintain robust stability as judged by these conditions. 
In this way, our optimization problem becomes a standard generalized eigenvalue problem, which can be then solved using GEVP technique. From this discussion, we have the following Remark 2.9.

Remark 2.9. Theorem 2.3 provides delay-dependent asymptotic stability criteria for the T-S fuzzy systems with an interval time-varying delay (2.4) in terms of solvability of LMIs [28]. Based on them, we can obtain the maximum allowable delay bound (MADB) $0 \leq h_{1} \leq h(t) \leq$ $h_{2}$ such that (2.4) is stable by solving the following convex optimization problem:

$$
\begin{array}{lc}
\text { Maximize } & h_{2} \\
\text { Subject to Theorem 2.3(Corollary 2.4) }
\end{array}
$$

Inequality (2.34) is a convex optimization problem and can be obtained efficiently using the MATLAB LMI Toolbox.

About how to seek an appropriate $\alpha$ satisfying $0<\alpha<1$, such that the upper bound $h$ of delay $h(t)$ subjecting to (2.29) is maximal, we give an algorithm as follows.

Algorithm 2.10 ((Maximizing $h>0)$ ). Step 1: For given $h_{d}$, choose an upper bound on $h$ satisfying (2.29), and then select this upper bound as the initial value $h_{0}$ of $h$.

Step 2: Set appropriate step lengths, $h_{\text {step }}$ and $\alpha_{\text {step }}$, for $h$ and $\alpha$, respectively. Set $k$ as a counter, and choose $k=1$. Meanwhile, let $h=h_{0}+h_{\text {step }}$ and the initial value $\alpha_{0}$ of $\alpha$ equals to $\alpha_{\text {step. }}$

5.

Step 3: Let $\alpha=k \alpha_{\text {step }}$, if inequality (2.29) is feasible, go to Step 4; otherwise, go to Step

Step 4: Let $h_{0}=h, \alpha_{0}=\alpha, k=1$ and $h=h_{0}+h_{\text {step }}$, go to step 3 .

Step 5: Let $k=k+1$. If $k \alpha_{\text {step }}<1$, then go to step 3; otherwise, stop.

Remark 2.11. For Algorithm 2.10, the final $h_{0}$ is the desired maximum of the upper bound of delay $h(t)$ satisfying (2.29) and $\alpha_{0}$ is the corresponding value of $\alpha$.

\section{Illustrative Examples}

To illustrate the usefulness of our results, this section will provide numerical examples. It will be shown that the proposed results can provide less conservative results that recent ones have given $[3,5-7,9,10,12,13,15,17-21,23,27]$. It is worth pointing out that our criteria carried out more efficiently for computation.

Example 3.1. Consider a time-delayed fuzzy system without controlling input. The T-S fuzzy model of this fuzzy system is of the following form.

Plant rules:

Rule 1: If $x_{1}(t)$ is $M_{1}$, then

$$
\dot{x}(t)=A_{1} x(t)+A_{d 1} x(t-h(t)) .
$$


Rule 2: If $x_{1}(t)$ is $M_{2}$, then

$$
\dot{x}(t)=A_{2} x(t)+A_{d 2} x(t-h(t))
$$

and the membership functions for rule 1 and rule 2 are

$$
M_{1}\left(x_{1}(t)\right)=\left(1-\frac{1}{1+e^{-5\left(x_{1}(t)-(\pi / 6)\right)}}\right) \frac{1}{1+e^{-5\left(x_{1}(t)-(\pi / 6)\right)}}, \quad M_{2}\left(x_{1}(t)\right)=1-M_{1}\left(x_{1}(t)\right),
$$

where

$$
A_{1}=\left[\begin{array}{cc}
-2 & 0 \\
0 & -0.9
\end{array}\right], \quad A_{d 1}=\left[\begin{array}{cc}
-1 & 0 \\
-1 & -1
\end{array}\right], \quad A_{2}=\left[\begin{array}{cc}
-1.5 & 1 \\
0 & -0.75
\end{array}\right], \quad A_{d 2}=\left[\begin{array}{cc}
-1 & 0 \\
1 & -0.85
\end{array}\right] .
$$

Solution 3.2. For system (3.1) and (3.2), by taking the parameter $h_{d}=0$ and $\alpha=0.6$, we get the Corollary 2.5 which remains feasible for any delay time $h \leq 2.2459$. In case of maximum allowable delay bound (MADB) $h=2.2459$, solving Corollary 2.5 yields the following set of feasible solutions:

$$
\begin{aligned}
& P=\left[\begin{array}{cc}
33.2342 & 7.9992 \\
7.9992 & 28.4879
\end{array}\right], \quad Q_{1}=\left[\begin{array}{cc}
0.0039 & -0.0007 \\
-0.0007 & 0.0029
\end{array}\right], \quad Q_{2}=\left[\begin{array}{cc}
78.8437 & -2.5471 \\
-2.5471 & 22.8291
\end{array}\right], \\
& R=\left[\begin{array}{cc}
1.6280 & 4.5093 \\
4.5093 & 13.7942
\end{array}\right], \quad X_{11}=\left[\begin{array}{ll}
1.8933 & 1.5877 \\
1.5877 & 8.4013
\end{array}\right], \quad X_{12}=\left[\begin{array}{cc}
0.1663 & -3.3981 \\
-3.4381 & -6.7700
\end{array}\right] \text {, } \\
& X_{13}=\left[\begin{array}{cc}
-1.2051 & -3.3469 \\
-3.3454 & -10.2327
\end{array}\right], \quad X_{22}=\left[\begin{array}{ll}
2.0259 & 1.5108 \\
1.5108 & 8.4337
\end{array}\right], \quad X_{23}=\left[\begin{array}{cc}
1.2030 & 3.3488 \\
3.3473 & 10.2310
\end{array}\right] \text {, } \\
& X_{33}=\left[\begin{array}{cc}
1.6246 & 4.5097 \\
4.5097 & 13.7915
\end{array}\right], \quad Y_{11}=\left[\begin{array}{ll}
5.1299 & 0.4116 \\
0.4116 & 5.9858
\end{array}\right], \quad Y_{12}=\left[\begin{array}{cc}
-0.1102 & -0.1624 \\
-0.1588 & -0.5302
\end{array}\right] \text {, } \\
& Y_{13}=\left[\begin{array}{ll}
0.1971 & 0.5872 \\
0.5915 & 1.7945
\end{array}\right], \quad Y_{22}=\left[\begin{array}{ll}
0.2962 & 0.6522 \\
0.6522 & 2.0458
\end{array}\right], \quad Y_{23}=\left[\begin{array}{ll}
0.3456 & 0.8055 \\
0.8058 & 2.5092
\end{array}\right], \\
& Y_{33}=\left[\begin{array}{ll}
0.8093 & 2.1141 \\
2.1141 & 6.4977
\end{array}\right] \text {. }
\end{aligned}
$$

Applying the criteria in $[9,10,12,17]$ and in this paper, the maximum values of $h$ for the stability of system under considerations are listed in Table 1. It is easy to see that the stability criterion in this paper gives a much less conservative result than the ones in $[9,10,12,17]$.

Furthermore, by taking the various $h_{1}\left(h_{d}=0.5\right)$, and from Theorem 2.3, we obtain the maximum allowable delay bound (MADB) $h_{2}$ as shown in Table 2 . From the above results of Table 2, if the $h_{1}$ increases, the delay time length increases. 
Table 1: Computation MADB $h$ for varying $h_{d}$ in Example 3.1.

\begin{tabular}{lcccccc}
\hline$h_{d}$ & 0.2 & 0.4 & 0.6 & 0.8 & 0.9 & $\geq 1$ \\
\hline Li et al. [9] & 1.0078 & 0.8164 & 0.6042 & 0.3356 & 0.1509 & Fail \\
Liu [12] & 1.0655 & 0.9247 & 0.8040 & 0.7306 & 0.7232 & 0.7232 \\
Lien [10] & 1.2052 & 1.0766 & 0.9589 & 0.8468 & 0.7968 & 0.7843 \\
Tian et al. [17] & 1.2682 & 1.2071 & 1.1565 & 1.1415 & 1.1415 & 1.1415 \\
Corollary 2.5 $(\alpha=0.6)$ & 2.0087 & 1.7944 & 1.5981 & 1.4114 & 1.3280 & 1.3071 \\
\hline
\end{tabular}

Table 2: MADB $h_{2}\left(h_{d}=0.5\right)$ for various $h_{1}$ in Example 3.1.

\begin{tabular}{lccccccc}
\hline$h_{1}$ & 0.1 & 0.3 & 0.5 & 0.7 & 0.9 & 1.0 & 2.0 \\
\hline Theorem 2.3 $(\alpha=0.6)$ & 1.3451 & 1.4016 & 1.4598 & 1.5208 & 1.5903 & 1.6357 & 2.1182 \\
\hline
\end{tabular}

Remark 3.3. Similar to Algorithm 2.10, an algorithm for seeking an appropriate $\alpha$ such that the upper bound of delay $0 \leq h_{1} \leq h(t) \leq h_{2}$, subjecting to (2.10a), (2.10b), (2.10c), and (2.10d) is maximal can be easily obtained.

Example 3.4. Consider a T-S fuzzy system with time-varying delay. The T-S fuzzy model of this fuzzy system is of the following form:

Rule 1: If $x_{1}(t)$ is $M_{1}$, then

$$
\dot{x}(t)=A_{1} x(t)+A_{d 1} x(t-h(t)) .
$$

Rule 2: If $x_{1}(t)$ is $M_{2}$, then

$$
\dot{x}(t)=A_{2} x(t)+A_{d 2} x(t-h(t)),
$$

and the membership functions for rule 1 and rule 2 are

$$
M_{1}(z(t))=\frac{1}{1+\exp \left(-2 x_{1}(t)\right)}, \quad M_{2}\left(x_{1}(t)\right)=1-M_{1}(z(t)),
$$

where

$$
A_{1}=\left[\begin{array}{cc}
-3.2 & 0.6 \\
0 & -2.1
\end{array}\right], \quad A_{d 1}=\left[\begin{array}{cc}
1 & 0.9 \\
0 & 2
\end{array}\right], \quad A_{2}=\left[\begin{array}{cc}
-1 & 0 \\
1 & -3
\end{array}\right], \quad A_{d 2}=\left[\begin{array}{cc}
0.9 & 0 \\
1 & 1.6
\end{array}\right] .
$$

Solution 3.5. By taking the parameter $h_{d}=0$, using Corollary 2.5, the maximum value of delay time for the System (3.6) and (3.7) to be asymptotically stable is $h \leq 1.0245$. By the criteria in $[9,16]$, the system (3.6) and (3.7) is asymptotically stable for $h \leq 0.58$ and $h \leq 0.6148$, respectively. Hence, for this example, the criteria proposed here significantly improve the estimate of the stability limit compared with the result of $[9,16]$. Employing the LMIs in $[18$ 21] and those in Corollary 2.5 yields upper bounds on $h_{2}$ that guarantee the stability of system (3.6) and (3.7) for various $h_{d}$, which are listed in Table 3, in which " - " means that the results 
Table 3: MADB $h$ for various $h_{d}$ in Example 3.4.

\begin{tabular}{lcccc}
\hline$h_{d}$ & 0.1 & 0.5 & 0.9 & $\geq 1$ \\
\hline Tian and Peng [18] & 0.3950 & 0.3950 & 0.3950 & - \\
Zuo and Wang [21] & 0.4808 & 0.4746 & 0.4454 & - \\
Wu and Li [19] & 0.4809 & 0.472 & 0.4455 & - \\
Z. Yang and Y. P. Yang [20] & 0.5030 & 0.4995 & 0.4988 & - \\
Kwon et al. [8] & 0.7236 & 0.7154 & 0.7014 & 0.7402 \\
Corollary 2.5 $(\alpha=0.6)$ & 0.8014 & 0.7908 & 0.7418 & - \\
\hline
\end{tabular}

are not applicable to the corresponding cases. It can be seen from Table 3 that Corollary 2.5 in this paper yields the least conservative stability test than other approaches, showing the advantage of the stability result in this paper.

Example 3.6. Consider a T-S fuzzy system with time-varying delay is of the following form: Plant rules.

Rule 1: If $x_{1}(t)$ is $M_{1}$, then

$$
\dot{x}(t)=A_{1} x(t)+A_{d 1} x(t-h(t)) .
$$

Rule 2: If $x_{1}(t)$ is $M_{2}$, then

$$
\dot{x}(t)=A_{2} x(t)+A_{d 2} x(t-h(t)),
$$

and the membership functions for rule 1 and rule 2 are

$$
M_{1}(z(t))=\frac{1}{1+\exp \left(-2 x_{1}(t)\right)}, \quad M_{2}\left(x_{1}(t)\right)=1-M_{1}(z(t)),
$$

where

$$
A_{1}=\left[\begin{array}{cc}
0.1 & 1 \\
0 & 0.2
\end{array}\right], \quad A_{d 1}=\left[\begin{array}{cc}
-0.3 & 2 \\
0 & -0.5
\end{array}\right], \quad A_{2}=\left[\begin{array}{cc}
0.1 & 2 \\
0 & -0.2
\end{array}\right], \quad A_{d 2}=\left[\begin{array}{cc}
-0.34 & 2 \\
0.01 & -0.4
\end{array}\right] .
$$

Solution 3.7. By using Corollary 2.4, the maximum allowable delay bound (MADB) can be calculated as $h_{2}=2.3725$. The results for stability conditions in different methods are compared in Table 4. It can be seen that the delay-dependent stability condition in this paper is less conservative than earlier reported ones in the literature $[5,7,10,15,18,27]$. Compared with Guan and chen [7] who used 5 LMI variables, Yoneyama [27] employed 20 LMI variables to get better stability results. To obtain improved stability results than those in $[15,18,27]$, we need 8 variables in Corollary 2.4 the same as $[5,10]$. It is also seen from Table 4 that the larger $r$ is the more unknown LMI variables are required in $[18,27]$. However, the unknown number of LMI variables is independent of $r$ in the results of this paper. It can be shown that the delay-dependent stability condition in this paper is the best performance. 
Table 4: MADB $h_{2}$ for unknown $h_{d}\left(h_{1}=0\right)$ in Example 3.6 for different methods (Number of variables: $N_{v}$; Number of fuzzy rules: $r$ ).

\begin{tabular}{lcc}
\hline Method & $h_{2}$ & $N_{v}$ \\
\hline Guan and Chen [7] & 1,2214 & 5 \\
Yoneyama [27] & 1.8294 & $8+6 r$ \\
Tian andPeng [18] & 1.9187 & $8+8 r$ \\
Lien [10] & 1.9187 & 8 \\
Chen et al. [5] & 1.9187 & 8 \\
Peng et al. [15] & 2.2812 & 15 \\
Corollary 2.4 $(\alpha=0.6)$ & 2.3725 & 8 \\
\hline
\end{tabular}

Remark 3.8. Similar to Algorithm 2.10, we can also find an appropriate scalar $\alpha$, such that the upper bound of delay $0 \leq h_{1} \leq h(t) \leq h_{2}$, subjecting to (2.26a), (2.26b), (2.26c), and (2.26d) reaches the maximum.

Example 3.9. Consider a nominal time-delay fuzzy system. The T-S fuzzy model of this fuzzy system is of the following form.

Rule 1: If $x_{1}(t)$ is $M_{1}$, then

$$
\dot{x}(t)=A_{1} x(t)+A_{d 1} x(t-h(t)) .
$$

Rule 2: If $x_{1}(t)$ is $M_{2}$, then

$$
\dot{x}(t)=A_{2} x(t)+A_{d 2} x(t-h(t)),
$$

and the membership function for rules 1 and 2 are

$$
M_{1}(z(t))=\frac{1}{1+\exp \left(-2 x_{1}(t)\right)}, \quad M_{2}\left(x_{1}(t)\right)=1-M_{1}(z(t))
$$

where

$$
A_{1}=\left[\begin{array}{cc}
-2.1 & 0.1 \\
-0.2 & -0.9
\end{array}\right], \quad A_{d 1}=\left[\begin{array}{cc}
-1.1 & 0.1 \\
-0.8 & -0.9
\end{array}\right], \quad A_{2}=\left[\begin{array}{cc}
-1.9 & 0 \\
-1.1 & -1.1
\end{array}\right], \quad A_{d 2}=\left[\begin{array}{cc}
-0.9 & 0 \\
-1.1 & -1.2
\end{array}\right] .
$$

Solution 3.10. The objective is to determine the maximum value of constant time-delay $h=$ $h_{2}\left(h_{1}=0\right)$ for which the system is stable. Table 5 compares works based on common quadratic functionals [7, 18-20,23] with the fuzzy functional of Corollary 2.4. It is clear by inspecting Table 5 that Corollary 2.4 provides the largest time delays. For comparison, Table 6 also lists the maximum allowable delay bound (MADB) $h$ obtained from the criterion [3]. It is clear that Corollary 2.5 gives much better results than those obtained by [3]. It is illustrated that the proposed stability criteria are effective in comparison to earlier and newly published results existing in the literature. 
Table 5: MADB $h=h_{2}\left(h_{1}=0\right)$ for different methods in Example 3.9.

\begin{tabular}{lcccccc}
\hline Method & {$[7]$} & {$[18]$} & {$[19]$} & {$[20]$} & {$[23]$} & Corollary 2.4 $(\alpha=0.6)$ \\
\hline$h$ & 1.25 & 1.85 & 3.37 & 3.85 & 4.61 & 5.4873 \\
\hline
\end{tabular}

Table 6: MADB $h$ for different $h_{d}$ for Example 3.9.

\begin{tabular}{lccccc}
\hline$h_{d}$ & 0.1 & 0.3 & 0.5 & 0.7 & 1.1 \\
\hline An and Wen [3] & 3.036 & 2.240 & 1.747 & 1.523 & 1.497 \\
Corollary 2.5 $(\alpha=0.6)$ & 4.4274 & 3.2378 & 2.5016 & 1.9494 & 1.5926 \\
\hline
\end{tabular}

Example 3.11. Consider a nominal time-delay fuzzy system. The T-S fuzzy model of this fuzzy system is of the following form.

Rule 1: If $x_{1}(t)$ is $M_{1}$, then

$$
\dot{x}(t)=A_{1} x(t)+A_{d 1} x(t-h(t)) .
$$

Rule 2: If $x_{1}(t)$ is $M_{2}$, then

$$
\dot{x}(t)=A_{2} x(t)+A_{d 2} x(t-h(t)),
$$

and the membership functions for rule 1 and rule 2 are

$$
M_{1}(z(t))=\sin ^{2}\left(x_{1}(t)\right), \quad M_{2}\left(x_{1}(t)\right)=\cos ^{2}\left(x_{1}(t)\right),
$$

where

$$
A_{1}=\left[\begin{array}{cc}
0 & 1 \\
-8 & -6
\end{array}\right], \quad A_{2}=\left[\begin{array}{cc}
0 & 1 \\
-8 & 0
\end{array}\right], \quad A_{d 1}=A_{d 2}=\left[\begin{array}{cc}
0 & 0 \\
1 & -2
\end{array}\right]
$$

Solution 3.12. Considering a constant time delay, by using Corollary 2.4, the maximum allowable delay bound (MADB) can be calculated as $h_{2}=0.4130\left(h_{1}=0, \alpha=0.5\right)$. By the criteria in $[13,23]$, the systems (3.17) and (3.18) is asymptotically stable for any $h$ that satisfies $h \leq 0.322$ and $h \leq 0.4060$, respectively. Hence, for this example, the criteria proposed here significantly improve the estimate of the stability limit compared with the result of $[13,23]$.

\section{Conclusion}

In this paper, we have dealt with the stability problem for T-S fuzzy systems with interval time-varying delay. By constructing a Lyapunov-Krasovskii functional, the supplementary requirement that the time derivative of time-varying delays must be smaller than one is released in the proposed delay-range-dependent stability criterion. By developing a delay decomposition approach, the information of the delayed plant states can be taken into full consideration, and new delay-range-dependent sufficient stability criteria are obtained in 
terms of linear matrix inequalities (LMIs) which can be easily solved by various optimization algorithms. Since the delay term is concerned more exactly, it is less conservative and more computationally efficient than those obtained from existing methods. Thus, the present method could largely reduce the computational burden in solving LMIs. Both theoretical and numerical comparisons have been provided to show the effectiveness and efficiency of the present method. Numerical examples are given to illustrate the effectiveness of our theoretical results.

\section{References}

[1] T. Takagi and M. Sugeno, "Fuzzy identification of systems and its application to modeling and control," IEEE Transactions Systems Man Cybernetics, vol. 15, pp. 16-132, 1985.

[2] K. Tanaka and M. Sugeno, "Stability analysis and design of fuzzy control systems," Fuzzy Sets and Systems, vol. 45, no. 2, pp. 135-156, 1992.

[3] J. Y. An and G. Wen, "Improved stability criteria for time-varying delayed T-S fuzzy systems via delay partitioning approach," Fuzzy Sets and Systems, vol. 185, no. 1, pp. 83-94, 2011.

[4] B. Chen and X. Liu, "Fuzzy guaranteed cost control for nonlinear systems with time-varying delay," IEEE Transactions on Fuzzy Systems, vol. 13, no. 2, pp. 238-249, 2005.

[5] B. Chen, X. P. Liu, and S. C. Tong, "Delay-dependent stability analysis and control synthesis of fuzzy dynamic systems with time delay," Fuzzy Sets and Systems, vol. 157, no. 16, pp. 2224-2240, 2006.

[6] B. Chen, X. P. Liu, and S. C. Tong, "New delay-dependent stabilization conditions of T-S fuzzy systems with constant delay," Fuzzy Sets and Systems, vol. 158, no. 20, pp. 2209-2224, 2007.

[7] X. P. Guan and C. L. Chen, "Delay-dependent guaranteed cost control for T-S fuzzy systems with time delays," IEEE Transactions on Fuzzy Systems, vol. 12, no. 2, pp. 236-249, 2004.

[8] O. M. Kwon, M. J. Park, S. M. Lee, and J. H. Park, "Augmented Lyapunov-Krasovskii functional approaches to robust stability criteria for uncertain Takagi-Sugeno fuzzy systems with time-varying delays," Fuzzy Sets and Systems, vol. 201, pp. 1-19, 2012.

[9] C. G. Li, H. J. Wang, and X. F. Liao, "Delay-dependent robust stability of uncertain fuzzy systems with time-varying delays," IEE Proceedings Control Theory \& Applications, vol. 151, no. 4, pp. 417-421, 2004.

[10] C. H. Lien, "Further results on delay-dependent robust stability of uncertain fuzzy systems with timevarying delay," Chaos, Solitons and Fractals, vol. 28, no. 2, pp. 422-427, 2006.

[11] P. L. Liu, "Robust exponential stability for uncertain time-varying delay systems with delay dependence," Journal of the Franklin Institute, vol. 346, no. 10, pp. 958-968, 2009.

[12] X. M. Liu, "Delay-dependent $H_{\infty}$ control for uncertain fuzzy systems with time-varying delays," Nonlinear Analysis, vol. 68, no. 5, pp. 1352-1361, 2008.

[13] L. A. Mozelli, F. O. Souza, and R. M. Palhares, "A new discretized Lyapunov-Krasovskii functional for stability analysis and control design of time-delayed TS fuzzy systems," International Journal of Robust and Nonlinear Control, vol. 21, no. 1, pp. 93-105, 2011.

[14] C. Peng, Y. C. Tian, and E. G. Tian, "Improved delay-dependent robust stabilization conditions of uncertain T-S fuzzy systems with time-varying delay," Fuzzy Sets and Systems, vol. 159, no. 20, pp. 2713-2729, 2008.

[15] C. Peng, L. Y. Wen, and J. Q. Yang, “On delay-dependent robust stability criteria for uncertain T-S fuzzy systems with interval time-varying delay," International Journal of Fuzzy Systems, vol. 13, no. 1, pp. 35-44, 2011.

[16] J. Qiu and J. H. Zhang, "New robust stability criterion for uncertain fuzzy systems with fast timevarying delays," Fuzzy Systems and Knowledge Discovery Lecture Notes in Computer Science, vol. 4223, pp. 41-44, 2006.

[17] E. Tian, D. Yue, and Y. Zhang, "Delay-dependent robust $H_{\infty}$ control for T-S fuzzy system with interval time-varying delay," Fuzzy Sets and Systems, vol. 160, no. 12, pp. 1708-1719, 2009.

[18] E. G. Tian and C. Peng, "Delay-dependent stability analysis and synthesis of uncertain T-S fuzzy systems with time-varying delay," Fuzzy Sets and Systems, vol. 157, no. 4, pp. 544-559, 2006.

[19] H. N. Wu and H. X. Li, "New approach to delay-dependent stability analysis and stabilization for continuous-time fuzzy systems with time-varying delay," IEEE Transactions on Fuzzy Systems, vol. 15, no. 3, pp. 482-493, 2007. 
[20] Z. Yang and Y.-P. Yang, "New delay-dependent stability analysis and synthesis of T-S fuzzy systems with time-varying delay," International Journal of Robust and Nonlinear Control, vol. 20, no. 3, pp. 313$322,2010$.

[21] Z. Zuo and Y. Wang, "Robust stability and stabilisation for nonlinear uncertain time-delay systems via fuzzy control approach," IET Control Theory \& Applications, vol. 1, no. 1, pp. 422-429, 2007.

[22] K. Gu, V. Kharitonov, and J. Chen, Stability of Time-Delay Systems, Birkhuser, Boston, Mass, USA, 2003.

[23] F. O. Souza, L. A. Mozelli, and R. M. Palhares, "On stability and stabilization of T-S fuzzy time-delayed systems," IEEE Transactions on Fuzzy Systems, vol. 17, no. 6, pp. 1450-1455, 2009.

[24] H. G. Zhang, Z. W. Liu, G. B. Huang, and Z. S. Wang, "Novel weighting-delay-based stability criteria for recurrent networks with time-varying delay," IEEE Transactions on Neural Networks, vol. 21, no. 1, pp. 91-106, 2010.

[25] X. L. Zhu and G. H. Yang, "Jensen integral inequality approach to stability analysis of continuoustime systems with time-varying delay," IET Control Theory \& Applications, vol. 2, no. 6, pp. 524-534, 2008.

[26] X.-L. Zhu and G.-H. Yang, "New results of stability analysis for systems with time-varying delay," International Journal of Robust and Nonlinear Control, vol. 20, no. 5, pp. 596-606, 2010.

[27] J. Yoneyama, "Robust stability and stabilization for uncertain Takagi-Sugeno fuzzy time-delay systems," Fuzzy Sets and Systems, vol. 158, no. 2, pp. 115-134, 2007.

[28] S. Boyd, L. E. Ghaoui, E. Feron, and V. Balakrishnan, Linear Matrix Inequalities in System and Control Theory, SIAM, Philadelphia, PA, USA, 1994. 


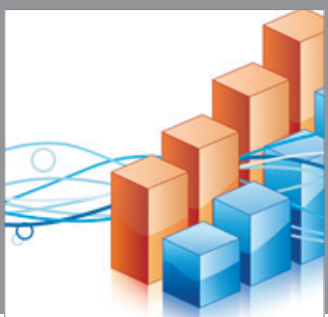

Advances in

Operations Research

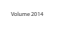

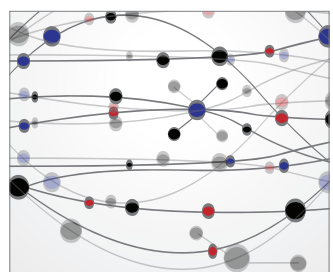

\section{The Scientific} World Journal
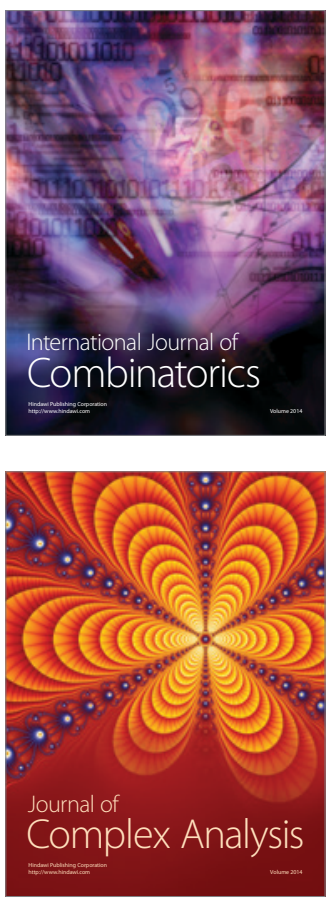

International Journal of

Mathematics and

Mathematical

Sciences
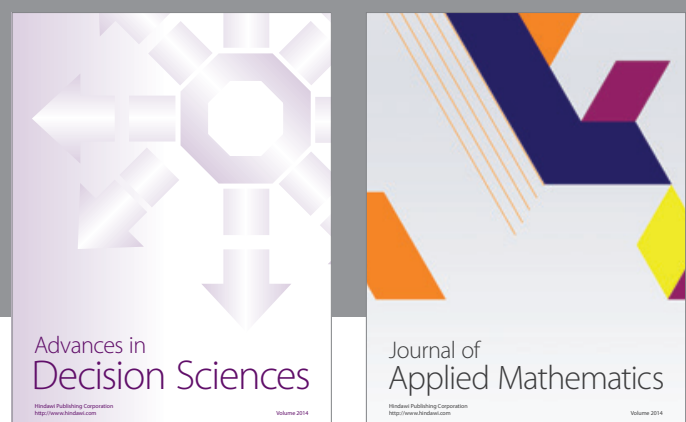

Journal of

Applied Mathematics
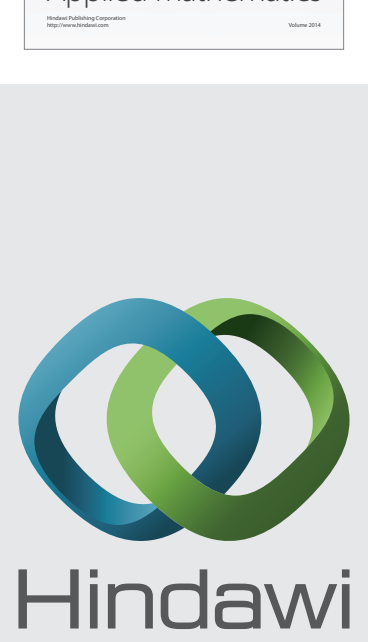

Submit your manuscripts at http://www.hindawi.com
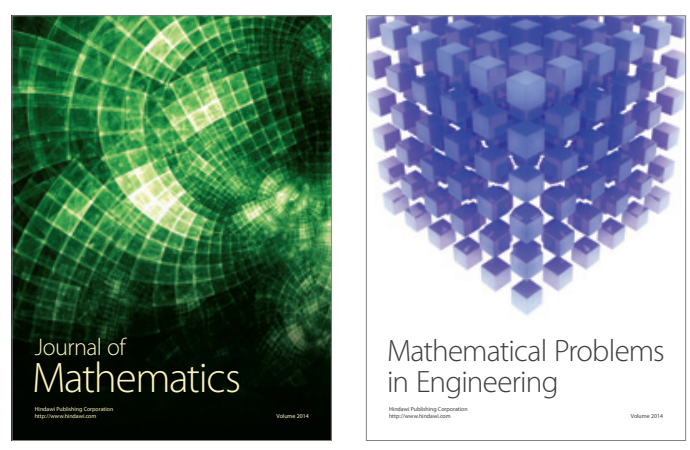

Mathematical Problems in Engineering
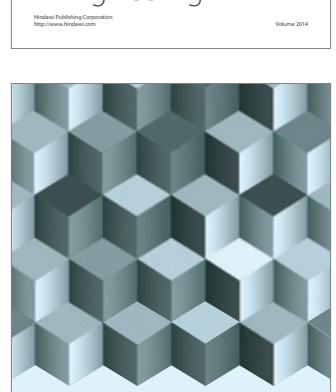

Journal of

Function Spaces
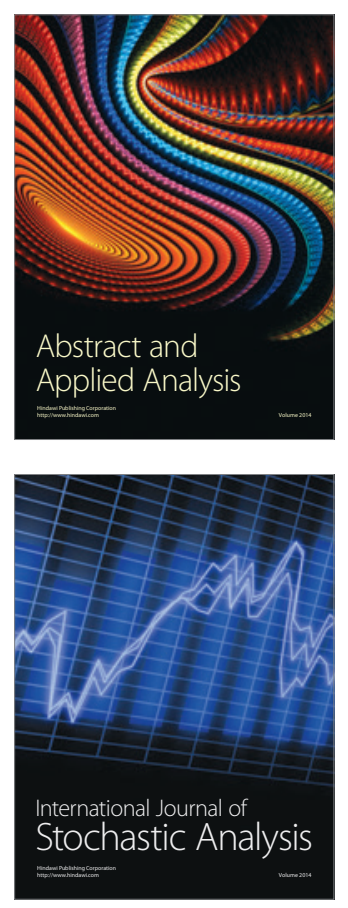

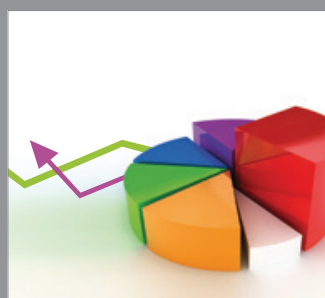

ournal of

Probability and Statistics

Promensencen
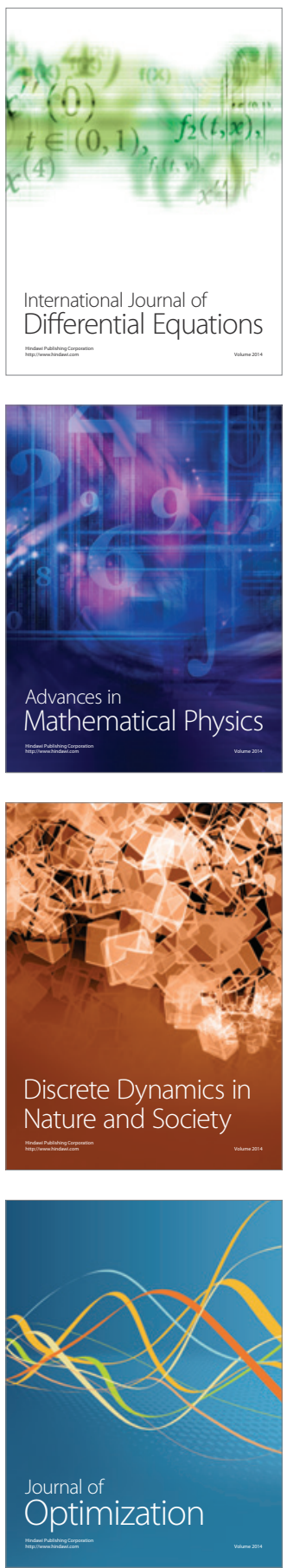